\title{
BMJ Open PARENTS 2 study protocol: pilot of Parents' Active Role and ENgagement in the review of Their Stillbirth/perinatal death
}

\author{
Danya Bakhbakhi, ${ }^{1}$ Dimitrios Siassakos, ${ }^{1}$ Claire Storey, ${ }^{2}$ Alexander Heazell, ${ }^{3,4}$ \\ Mary Lynch, ${ }^{1}$ Laura Timlin, ${ }^{1}$ Christy Burden ${ }^{1}$
}

To cite: Bakhbakhi D, Siassakos D, Storey C, et al. PARENTS 2 study protocol: pilot of Parents' Active Role and ENgagement in the review of Their Stillbirth/ perinatal death. BMJ Open 2018;8:e020164. doi:10.1136/ bmjopen-2017-020164

- Prepublication history and additional material for this paper are available online. To view these files, please visit the journal online (http://dx.doi. org/10.1136/bmjopen-2017020164).

Received 19 0ctober 2017 Revised 17 November 2017 Accepted 24 November 2017

CrossMark

${ }^{1}$ Centre for Academic Women's Health, University of Bristol, Women's Health, Southmead Hospital, Bristol, UK

${ }^{2}$ International Stillbirth Alliance, Bristol, UK

${ }^{3}$ Maternal and Fetal Health Research Centre, School of Medical Sciences, Faculty of Biological, Medicine and Health, University of Manchester, Manchester, UK

${ }^{4}$ Central Manchester University Hospitals NHS Foundation Trust, Manchester Academic Health Science Centre, St Mary's Hospital, Manchester, UK

Correspondence to Dr Danya Bakhbakhi; db12202@bristol.ac.uk

\section{ABSTRACT}

Background The perinatal mortality review meeting that takes place within the hospital following a stillbirth or neonatal death enables clinicians to learn vital lessons to improve care for women and their families for the future. Recent evidence suggests that parents are unaware that a formal review following the death of their baby takes place. Many would welcome the opportunity to feedback into the meeting itself. Parental involvement in the perinatal mortality review meeting has the potential to improve patient satisfaction, drive improvements in patient safety and promote an open culture within healthcare. Yet evidence on the feasibility of involving bereaved parents in the review process is lacking. This paper describes the protocol for the Parents' Active Role and Engangement iN the review of their Stillbirth/perinatal death study (PARENTS 2) , whereby healthcare professionals' and stakeholders' perceptions of parental involvement will be investigated, and parental involvement in the perinatal mortality review will be piloted and evaluated at two hospitals.

Methods and analysis We will investigate perceptions of parental involvement in the perinatal mortality review process by conducting four focus groups. A three-round modified Delphi technique will be employed to gain a consensus on principles of parental involvement in the perinatal mortality review process. We will use three sequential rounds, including a national consensus meeting workshop with experts in stillbirth, neonatal death and bereavement care, and a two-stage anonymous online questionnaire. We will pilot a new perinatal mortality review process with parental involvement over a 6 -month study period. The impact of the new process will be evaluated by assessing parents' experiences of their care and parents' and staff perceptions of their involvement in the process by conducting further focus groups and using a Parent Generated Index questionnaire.

Ethics and dissemination This study has ethical approval from the UK Health Research Authority. We will disseminate the findings through national and international conferences and international peer-reviewed journals.

\section{INTRODUCTION}

In 2015, the perinatal mortality rate (stillbirths and deaths under 7 days)in the UK was 6.5 in
Strengths and limitations of this study

- Although patients have been previously involved in healthcare improvement, the involvement of parents in such a sensitive area as the review of perinatal deaths would be a genuinely novel, yet urgently needed and recommended innovation for patient care.

- Investigation and implementation across two different and diverse sites across the UK increases the generalisability of the results.

- The relatively small sample size might limit to some extent the scope of the findings. Even so, the mixedmethods methodology allows for rich data with clear implications.

1000 births. ${ }^{1}$ The negative impact of stillbirth or neonatal death on parents, siblings and the wider family can be profound. ${ }^{2}$ Following a stillbirth or early neonatal death, a perinatal mortality review (PNMR) meeting with healthcare professionals should take place within the hospital. The purpose of the perinatal review process is multifaceted. First, it can help confirm the diagnosis and the events that led up to the death of the baby, in addition to acknowledgement of circumstances where care issues have been identified and where lessons can be learned to improve prevention and care in the future. ${ }^{3}$

The Morecambe Bay enquiry highlighted several incidents where substandard care led to poor outcomes for mothers and babies. The investigation recommended that these serious adverse events should be subject to a standardised, multidisciplinary peer review and that feedback from families should be sought. ${ }^{4}$ The UK government support these recommendations to facilitate safer maternity services. ${ }^{5}$ However, the 2015 Mothers and Babies: Reducing Risk through Audits and Confidential Enquires across the UK report 
showed that input from families is rarely sought. Only $5 \%$ (6 out of 133) cases had documented evidence that feedback had been obtained. ${ }^{6}$ The Royal College of Obstetricians and Gynaecologists' 'Each Baby Counts' report of intrapartum stillbirths, early neonatal deaths and severe brain injuries found a similar pattern. ${ }^{7}$ In a quarter of instances, parents were not made aware of a review taking place and only $28 \%$ of parents were invited to contribute if they wished. ${ }^{7}$

Evidence from a recent focus group of bereaved parents conducted by the authors of this protocol (the PARENTS 1 Study) indicated that parents were largely unaware that a review of their baby's death took place and found it distressing that they were not involved or kept informed. ${ }^{8}$ Parents were unanimously in favour of an optional opportunity to contribute information and would welcome a flexible system that could provide them with feedback, outcomes and lessons learned following the review. ${ }^{8}$

Parental involvement into the PNMR meeting could improve patient experience, drive improvements in patient safety and promote an open culture within the healthcare system. Involving patients in understanding the events they have experienced could potentially help in the healing process. Furthermore, it may result in cost savings; evidence has highlighted the significant far-ranging psychological and socioeconomic effect of perinatal death, including depression, problems with subsequent pregnancies and additional negative impact on society. ${ }^{9}$ This paper describes the protocol for the PARENTS 2 Study whereby healthcare professionals' and stakeholders' perceptions of parental involvement will be investigated, and parental involvement in the PNMR will be piloted and evaluated at two hospitals in the UK.

\section{METHODS AND ANALYSIS}

\section{Preintervention focus groups: staff and stakeholders}

\section{Participant selection}

The opinions of healthcare professionals and key stakeholders on the PNMR process will be sought, with emphasis on their perceptions of facilitators and barriers to parental input. We will undertake four focus groups: two at North Bristol NHS Trust (NBT; Bristol, UK) and two at Central Manchester Foundation Trust (CMFT; Manchester, UK). Five to 10 maternity healthcare professionals and 5 to 10 stakeholders will be recruited at each site for each focus group with aim to reach data saturation.

Participants will be purposively sampled to ensure a diverse range of staff and stakeholder viewpoints. The healthcare professional focus group will aim to include midwives, obstetric consultants, junior doctors, neonatology consultants, nursing staff and chaplaincy services. The stakeholder focus groups will include clinical directors, policy-makers and commissioners. Participants will be approached via email and face to face. The number of staff refusing to take part will be documented.

\section{Recruitment}

Healthcare professionals and stakeholders will be informed of The PARENTS 2 Study by an introductory email and information leaflet at each unit. The information leaflet will invite healthcare professionals and stakeholders to participate in a $90 \mathrm{~min}$ focus group and participants will be recruited by a research midwife.

\section{Focus group interviews}

The focus group will take place in a meeting room away from the clinical environment. Focus group attendees will be asked to provide written consent, including consent to be audio recorded and for anonymised quotes to be used in reporting. Researchers (the authors CB, DS, CS, ML) who are experienced in qualitative interviewing will conduct the focus group discussions. $\mathrm{CB}$ is a female postdoctorate research fellow (MD), DS is a male consultant senior lecturer (MD), ML is a female experienced bereavement research midwife and DB is a female clinical research fellow. CS was a female external research assistant who conducted interviews at both NBT and CMFT.

A research midwife and/or a research facilitator will also be present to cofacilitate the focus groups and moderate group dynamics. The researchers will follow the focus group schedule developed and piloted with the Project Advisory Board (see online supplementary file 1). Furthermore, field notes will be taken to give the interview context. The researchers and research midwife all work clinically within the hospital trusts and therefore may have already have a professional relationship with the participants. The participants will be made aware of the goals of the research prior to commencing the interviews. The following approach will be undertaken: familiarisation period and scene setting, discussion, checking of specific factors not discussed but thought to be important by the research group, opinions felt by participants and summarisation.

\section{Data analysis}

The audio recordings of the focus group interviews will be transcribed in full, stored and analysed using NVivo10 software. The focus group data will be analysed using the technique described by Braun and Clark ${ }^{10}$ called inductive thematic analysis. ${ }^{10}$ This is a six-stage process: familiarisation with the data; generation of initial codes; searching for themes; reviewing themes; defining themes and naming themes. The transcripts will be coded independently in duplicate, while the wider members of the research team will have the opportunity to read through each focus group transcript. The transcripts will also be sent to participants for comment. Emerging themes from the data will be discussed with the whole research team to enable cross-sectional analysis and agreement of themes. A coding tree will be produced from the resulting themes and subthemes with supporting quotes identified. Participants will also be given the opportunity to feedback on the findings. 
Findings from interim data analyses will be discussed in meetings of the Project Steering Committee. The purpose will be to identify procedural issues, finalise the analysis, establish the credibility and applicability of the results, triangulate with other sources of evidence and combine the findings with the previous PARENTS 1 study focus group. ${ }^{8}$

\section{Consensus on parental involvement}

A modified Delphi technique will be used to gain a national consensus parental involvement and the PNMR process. We will use three sequential rounds including a consensus meeting workshop and a two-stage anonymous questionnaire. A national panel of experts in stillbirth and bereavement care and patient advocates will be identified from the International Stillbirth Alliance, The Stillbirth and Neonatal Death Charity, Child Bereavement Care UK and Bliss UK charity. The expert group will also include a small subsample of clinical directors, clinical commissioners and department managers, lead obstetricians and neonatologists, lead midwives and neonatal nurses and the research team.

\section{Round 1}

A 5-hour consensus workshop will take place. The meeting will focus on four key areas including getting feedback from parents, the format of the PNMR meeting, the parental pathway and challenging aspects of involving parents. Themes and principles will be presented from the focus groups of parents and healthcare professionals to the panel of experts alongside current evidence in this area of bereavement care. The expert group will be asked to generate ideas and solutions specifically addressing the four key areas with the overall aim to create principles of how to implement a PNMR process with parental involvement. Each participant will be asked to submit their solutions to the moderator of the group (member of the research team) who will collate information to generate overarching principles for parental involvement.

\section{Round 2}

The expert panel from the consensus meeting will then be invited via a personalised email to complete an anonymous web-based survey (Smart Survey). The principles and survey will by piloted by the study committee prior to its usage to ensure readability and ease of completion. Participants will then rank the principles generated from round one using a 9-point Likert scale from 1 (not important) to 9 (critical). This scale was created by the Grading of Recommendations Assessment, Development and Evaluation working group and has been used widely in other consensus research studies. ${ }^{11}$ For each principle generated, the median and IQR will be summarised graphically. 'Consensus' will be reached if over $70 \%$ or participants score the principle as 'critical' (score 7 to 9) and less than 15\% of participants' score the principle as 'not important' (score 1 to 3). Principles will be excluded if $70 \%$ or participants score the principle as 'not important (score 1 to 3 ) and less than $15 \%$ of participants' score the principle as 'critical' (score 1 to 3). No consensus principles would be anything else not included in the ranges stated. Descriptive statistics will be used to analyse the results.

\section{Round 3}

Participants will be presented with the results of the web-based survey and they will be asked to rescore the principles where no consensus was reached. The final principles agreed will then be used for the pilot of parental involvement.

\section{Pilot intervention: parental input in the PNMR process} Participant selection

Women and their partners will be invited to participate in the study if they experience a perinatal death within the 6-month pilot of the intervention, in two diverse hospitals (NBT and CMFT). This will include all stillbirths (birth of a baby with no signs of life after 24 weeks completed gestation) and neonatal deaths (death of a baby within 4 weeks of birth). With an estimated perinatal death rate of about 1:200 and conservative estimates of recruitment rate of about $60 \%$ (both units have track record of $>60 \%$ $70 \%$ recruitment of bereaved parents in research), we estimate to recruit a pragmatic sample of about 10-12 parent sets at each site within the 6-month study period (7-8000 births per annum: $2 \times 60 \% \times 1: 200)$ (figure 1 ).

\section{Method of approach}

The midwife or neonatal nurse who has cared for the parent/s in hospital will initially approach bereaved parent/s on the day they are due to return to community after the perinatal death. The parent/s will be given a letter and a brief initial information leaflet about the study. The information leaflet will explain briefly what the research aims are, who the researchers are, what the timetable of events is during the research and that participation is voluntary. It will be emphasised that not taking part will not in any way influence the ongoing care the parent/s receive. Parent/s will be asked to indicate to their midwife or neonatal nurse, whether they agree to receive further information from the research team, using their preferred communication method (email or post).

\section{Recruitment}

An information pack will be sent to parent/s wishing to participate using the communication method of their choice within 1 week after the perinatal loss has occurred. The information pack will contain a cover letter, a detailed information leaflet about the study, a sample consent form, a PNMR parent feedback form, a Parent Generated Index questionnaire (PGI) and a proposed date for the PNMR meeting.

The exact PNMR parent feedback form will be determined from the modified Delphi consensus study. The form will allow parent/s to submit questions or comments which will be discussed at the multidisciplinary PNMR 


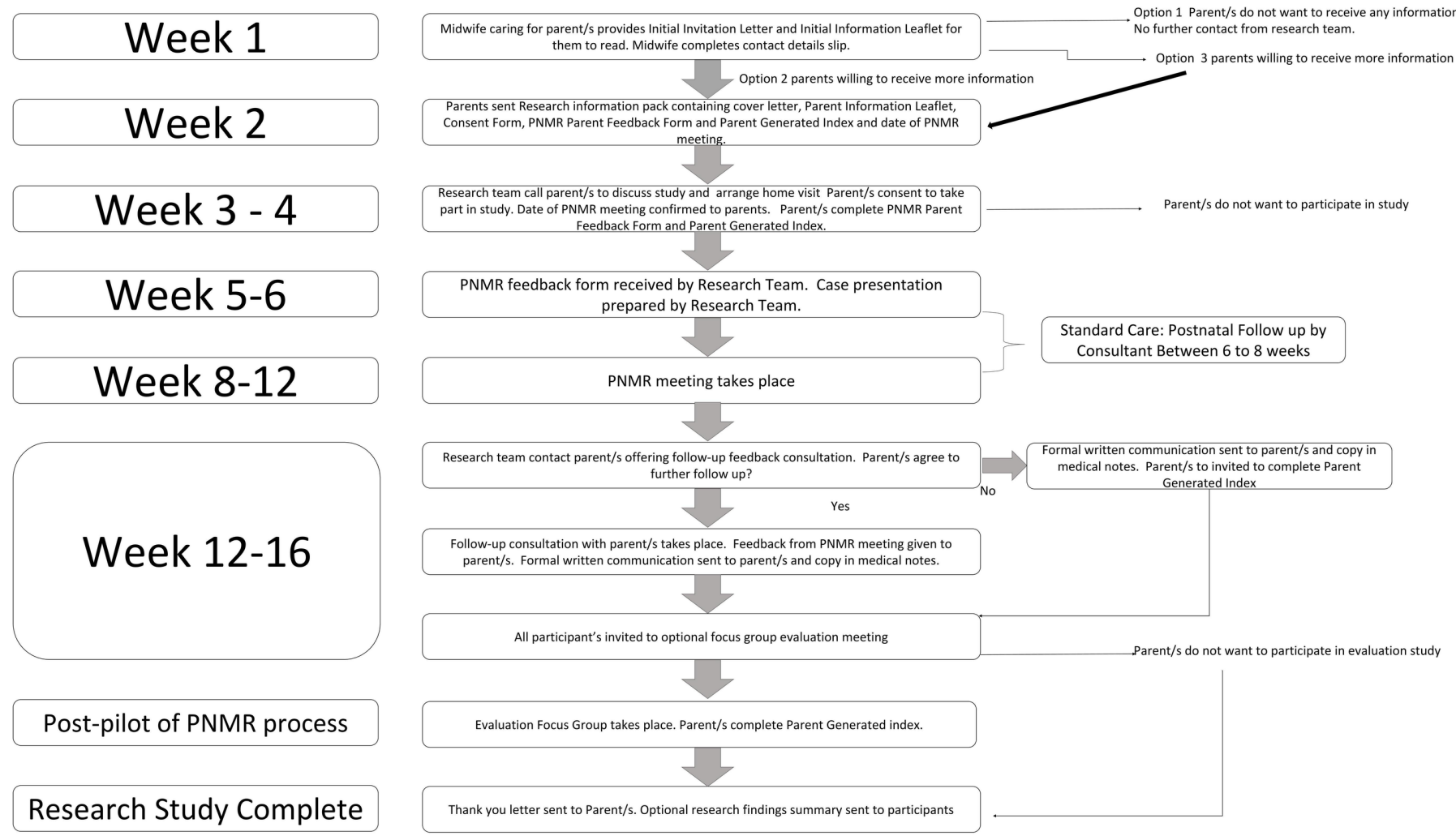

Figure 1 Flow chart of pilot perinatal mortality review (PNMR) process with parental involvement.

meeting. Data from the PARENTS 1 Study suggests that parent/s would like the form to be specific to the type of death that has occurred; they would like to be given the opportunity to give feedback to the hospital and community team; they would like the option to give positive and negative feedback about their care and they would like suggestions on what type of feedback they should give including clinical and non- clinical care. ${ }^{8}$

Parent/s will receive a telephone call from the research team 1 week after the information pack has been sent to them. At their chosen time, the researcher will enquire whether the parent/s have read and received the necessary information, address any questions they might have about the study. Once all questions have been fully answered, the researcher will record their verbal consent of their wish to participate and receive a home visit from the research midwife to gain formal consent. They will be encouraged to read and/or complete the PNMR feedback form prior to the home visit so the research midwife can address any questions they might have about the form during the home visit. The research midwife will then arrange a convenient time for them to have a home visit where the women and/or their partner will be consented to take part in the study. Participating parent/s will then be invited to complete the PNMR feedback form and a PGI.

The PGI is a validated antenatal and postnatal quality of life instrument in which the variables and scores are completely respondent driven. ${ }^{12}$ It allows participants to include quality of life issues that are important to them which are not captured by existing tools. Parents will be invited to complete the PGI before and after their involvement in the pilot PNMR process at NBT and CMFT for comparison.

The research midwife will then confirm the date and time of the PNMR meeting to them. They will also be asked their preferred method of correspondence for example, written, telephone or a face-to-face meeting following the PNMR meeting to discuss the findings of the review of their case. Parent/s will have in approximately 4 weeks to decide if they wish to participate and consent for the study.

During the recruitment home visit, they will also be invited to participate in a focus group of 5 to 10 other parents after completion of the pilot to discuss their experience of the PNMR process. A further opportunity to take part in this part of the study will be offered following the PNMR meeting.

\section{PNMR meeting}

The PNMR meeting will take place approximately to 8 to 12 weeks after the perinatal death at NBT or CMFT. All questions and comments received from parents via the feedback form will be discussed in a standardised format at the meeting. Two members of the research team will be present at the PNMR to facilitate the running of the meeting and to ensure parents' feedback is addressed. Obstetricians, midwives (hospital and community staff), neonatologists, pathologists and ultrasonographers will be invited to attend. Furthermore, those healthcare professionals specifically involved in the care of the parent/s discussed at the meeting, will be invited to attend the 
PNMR meeting; for example, community staff if appropriate. This will ensure that any specific parental feedback is adequately addressed by the member/s of staff who cared for them. If specific healthcare professionals are unable to attend, we will discuss the parent/s feedback with them prior to the meeting and request that they submit a written summary. This will then be discussed at the meeting. Should any parent submit any personal or upsetting feedback about a specific member of staff this will be dealt with in the usual complaint escalation process independent of the research project.

A member of the research team will prepare a presentation for each perinatal death case that has been consented to be involved in the study. This will follow a standardised format across both research sites where the parental medical history, antenatal, intrapartum and postnatal care and postmortem findings will be discussed. This clinical information is already discussed at the PNMR meetings at both sites. If parents do not consent to participate in the study, their case will be reviewed in the format already used by NBT and CMFT and their normal care will not be affected by the study.

All discussions that take place about the cases consented into the study will be audio recorded by one of the research team members. We will follow NBT and CMFT guidelines for audio recording. Members of staff will be consented at the start of the meeting and they will be asked to give permission for their comments to be audio recorded. Should a member of staff not consent to the study they will still be allowed to participate in the PNMR discussion but the audio recorder will be switched off when they are talking.

The research team members will ensure that each feedback comment on the PNMR parent feedback form is addressed with any salient learning points clearly documented on a PNMR outcome form. Furthermore, the PNMR meetings themselves will be observed with ethnography, and key challenges and successes of implementation of parental input into the meeting will be captured. We will use a 'rapid cycle evaluation' system, where we will aim to use 'single loop learning' to continuously refine the PNMR process using our learning points of the meeting. ${ }^{13} \mathrm{~A}$ member of the research team present at the meeting will record any challenges and successes of implementing parental input into the PNMR process. These recordings will then be reviewed by the research team after each meeting and the research team will seek to address any barriers prior to the next PNMR meeting.

\section{Feedback consultation}

Once the PNMR meeting has taken place, a further telephone call to the parent/s will be made by a member of the research team. Parent/s will be offered a face-to-face consultation, verbal telephone or postal feedback on the outcome of the PNMR meeting. A formal letter will be sent through post detailing the outcome of the meeting for those participants who consent to receive it, and a separate copy will be kept in the patient's medical notes.
Parent/s at this point will be asked if they would like to participate in a focus group on completion of the pilot of the PNMR process with parental input. Their interest and contact details will be documented in the research site file and they will be recontacted once the pilot is complete. Parent/s who do not wish to participate in focus group will be invited to complete a postintervention PGI, and this will be sent to them through post with a prepaid envelope to complete the questionnaire.

\section{Closing the process}

All participants will receive a thank you letter from the research team once the PNMR process and consultation meeting has taken place, sent within 2 weeks.

A summary letter about the research will be sent out to participants if they consent to receive this once the project has been completed. They will have been informed previously that this will not be available for about 1 year.

\section{Evaluation of parent and healthcare professionals experience of intervention}

\section{Evaluation of parent experience}

Following the 6-month pilot of parental involvement in the PNMR, all parents will be invited to take part in an evaluation focus group. The number of parents declining to participate will be documented. There will be one focus group composed of 5 to 10 parents at NBT and one focus group composed of 5 to 10 parents at CMFT. The focus groups will discuss parents' perceptions of their inclusion in the PNMR process and will aim to ascertain what went well and what might be improved and how (see online supplementary file 2). Parents' experience of care will again be evaluated with the PGI, and this will be compared with the results from the preintervention questionnaire. ${ }^{12}$

Parents will be recruited by the research midwife who will contact them via telephone to ascertain if they wish to participate in the focus group. Parents will have been initially informed of the evaluation of the study in the recruitment stage of the pilot of the intervention. If they consent they will be invited to a 90 min focus group with other parents who have participated in the study.

\section{Evaluation of healthcare professionals experience}

We will undertake one focus group of 5 to 10 healthcare professionals at NBT and one focus group of 5 to 10 healthcare professionals at CMFT. We will assess healthcare professionals' experience of being involved in a PNMR process with parental involvement. We will try to ascertain what went well and what could be improved for future implementation (see online supplementary file 3 ). Participants will be purposively sampled to ensure a diverse range of staff who have been involved in the PNMR process. The healthcare professional focus group will include midwives, obstetric consultants, junior doctors, neonatology consultants, nursing staff and chaplaincy services. The number of staff declining to take part will be documented. 


\section{Focus group interviews}

The focus group will take place in a meeting room away from the clinical environment. Focus group attendees will be asked to provide written consent, including consent to be audio recorded and for anonymised quotes to be used in reporting. The study researchers (the authors CB, DS, CS, ML) will again conduct the focus group discussions. A research midwife and/or a research facilitator will also be present to cofacilitate the focus groups and moderate group dynamics. The researchers will follow the focus group schedules piloted and tested with the Project Advisory Board (see online supplementary files 2 and 3). Furthermore, field notes will be taken to give the interview context. The researchers and research midwife all work clinically within the hospital trusts and therefore may have already have a professional relationship with the participants. The participants will be made aware of the goals of the research prior to commencing the interviews. The following approach will be undertaken: familiarisation period and scene setting, discussion, checking of specific factors not discussed but thought to be important by the research group, opinions felt by participants and summarisation. The qualitative researcher will also keep field notes to facilitate interpreting the data in context.

\section{Data analysis}

The audio recordings of the focus group interviews will be transcribed in full, stored and analysed using NVivo10 software. The focus group data will again be analysed using inductive thematic analysis. ${ }^{10}$ The transcripts will be coded independently in duplicate, while the wider members of the research team will have the opportunity to read through each focus group transcript. The transcripts will also be sent to participants for comment. Emerging themes from the data will be discussed with the whole research team to enable cross-sectional analysis and agreement of themes. A coding tree will be produced from the resulting themes and subthemes with supporting quotes identified. Participants will also be given the opportunity to feedback on the findings. Furthermore, findings from interim data analyses will be discussed in meetings of the Project Steering Committee.

\section{Ethics and dissemination}

Ethics

This study has ethical approval from the UK Health Research Authority and the date of approval is 30 May 2017. This is an ongoing study taking place from the 30 May 2017 until 1 February 2018.

\section{Dissemination}

The results of our study will be presented at a national and international level by submitting to the conferences of the Royal College of Obstetricians and Gynaecologists, the Royal College of Midwives, the Neonatal Society, the annual joint meeting of the British Maternal and Fetal Medicine Society with the British Association of Perinatal Medicine, the annual conference of the International
Stillbirth Alliance and through Health Foundation links and events. Furthermore, we will disseminate our work and engage our peers in implementation of the improved PNMR process by publishing in a high-impact factor peer-reviewed journal.

We endeavour to inform and influence national and international policy through established stillbirth networks, the Royal College of Obstetricians and Gynaecologists, the Department of Health and the International Stillbirth Alliance. The aim is to implement an improved PNMR process with parental input that can be rolled out across the UK and beyond.

Contributors DB drafted the manuscript and was involved revising the manuscript critically for important intellectual content. DS, CS, AH, ML, CB and LT were involved in revising the manuscript critically for important intellectual content. All authors read and approved the final manuscript. CB is the grant holder, DB is the principal investigator and DS is the chief investigator and guarantor.

Funding The study is funded by the Health Foundation. The consensus meeting is sponsored by Sands, the Stillbirth and Neonatal Death charity. DB receives salary support from the National Institute of Health Research (NIHR) Academic Clinical Fellowship. AH receives salary support from Tommy's and the NIHR Clinician Scientist Fellowship. CB receives salary support from the NIHR Academic Clinical Lectureship.

Disclaimer The views and opinions expressed herein do not necessarily reflect those of the NIHR, National Health Service or the Department of Health.

Competing interests None declared.

Ethics approval This study has ethical approval from the UK Health Research Authority (Research Ethics Committee reference no 17/WM/0123).

Provenance and peer review Not commissioned; externally peer reviewed.

Open Access This is an Open Access article distributed in accordance with the Creative Commons Attribution Non Commercial (CC BY-NC 4.0) license, which permits others to distribute, remix, adapt, build upon this work non-commercially, and license their derivative works on different terms, provided the original work is properly cited and the use is non-commercial. See: http://creativecommons.org/ licenses/by-nc/4.0/

(C) Article author(s) (or their employer(s) unless otherwise stated in the text of the article) 2018. All rights reserved. No commercial use is permitted unless otherwise expressly granted.

\section{REFERENCES}

1. National Statistics Office. Office for National Statistics: Stillbirth. https://www.ons.gov.uk/peoplepopulationandcommunity/birthsdea thsandmarriages/stillbirths (cited Jan 9 2017).

2. Burden C, Bradley S, Storey C, et al. From grief, guilt pain and stigma to hope and pride: a systematic review and meta-analysis of mixed-method research of the psychosocial impact of stillbirth. BMC Pregnancy Childbirth 2016;16:9.

3. National Quality Board. National Guidance on Learning from Deaths-NHS England. $2017 \mathrm{https} / / / w w w . e n g l a n d . n h s . u k / w p-$ content/uploads/2017/03/nqb-national-guidance-learning-fromdeaths.pdf (cited Oct 6 2017).

4. Kirkup B. The Report of the Morecambe Bay Investigation [Internet]. https://www.gov.uk/government/uploads/system/uploads/attachment data/file/408480/47487_MBI_Accessible_v0.1.pdf (cited 2017 Feb 5).

5. The Government Select Committee. The government response to the Freedom to Speak Up consultation, the Public Administration Select Committee report "Investigating Clinical Incidents in the NHS", and the Morecambe Bay Investigation.

6. MBRRACE. Perinatal Mortality Report, November 2015. available from. https://www.npeu.ox.ac.uk/downloads/files/mbrrace-uk/ reports/MBRRACE-. (cited Mar 1 2017).

7. Royal College of Obstetricians and Gynaecologists. Each Baby Counts: 2015 Summary Report.

8. Bakhbakhi D, Siassakos D, Burden C, et al. Learning from deaths: parents' active role and engagement in the review of their stillbirth/ perinatal death (the PARENTS 1 study). BMC Pregnancy Childbirth 2017;17:333. 
9. Heazell AE, Siassakos D, Blencowe H, et al. Stillbirths: economic and psychosocial consequences. Lancet 2016;387:604-16.

10. Braun V, Clarke V. Using thematic analysis in psychology. Qual Res Psychol 2006;3:77-101.

11. GRADE Working Group. GRADE [Internet]. http://www. gradeworkinggroup.org (cited Jan 9 2017).
12. Symon A, Downe S, Finlayson KW, et al. The feasibility and acceptability of using the Mother-Generated Index (MGI) as a Patient Reported Outcome Measure in a randomised controlled trial of maternity care. BMC Med Res Methodol 2015;15:100.

13. The Health Foundation. Evaluation: What to consider. Commonly asked questions about evaluation of quality improvement in health care. 2015. 